\title{
Infections respiratoires
}

\section{Respiratory infections}

C SRLF et Springer-Verlag France 2013

\section{EP014 \\ Pneumonies acquises sous ventilation mécanique chez les brûlés en réanimation \\ A. Hachani ${ }^{1}$, A. Mokline ${ }^{1}$, H. Fredj ${ }^{1}$, B. Gasri ${ }^{1}$, I. Rahmani ${ }^{1}$, H. Oueslati ${ }^{1}$, S. Tlaili ${ }^{1}$, R. Hammouda ${ }^{1}$, L. Gharsallah ${ }^{1}$, R. Aloui ${ }^{1}$, A. Ghanem ${ }^{2}$, A.A. Messadi ${ }^{1}$ \\ ${ }^{1}$ Réanimation des brûlés, centre de traumatologie et des grands brûlés, Tunis, Tunisie \\ ${ }^{2}$ Laboratoire de biologie clinique, centre de traumatologie et des grands brûlés, Tunis, Tunisie}

Introduction : La pneumonie acquise sous ventilation mécanique (PAVM) représente la première localisation d'infections nosocomiales en réanimation, compliquant 8 à $28 \%$ des sujets ventilés. Elle est grevée d'une lourde morbimortalité. En effet, elle allonge la durée de séjour de 22 jours en USI, et est la première cause de décès due à une infection nosocomiale. Le but de cette étude est d'évaluer les caractéristiques épidémiologiques, microbiologiques et pronostiques des PAVM chez les brûlés.

Patients et méthodes : étude rétrospective menée au service de réanimation des brûlés de Tunis sur une période de 12 mois (janvier 2012décembre 2012). Ont été inclus, les patients admis en réanimation pour des brûlures de la face et/ou des brûlures $>20 \%$ nécessitant le recours à la ventilation mécanique pendant plus de 48 heures. Ont été exclus, les sujets dont la durée de séjour est moins de $48 \mathrm{H}$ ou ceux considérés en dehors de toute ressources thérapeutique. Le diagnostic de PAVM est basé sur un infiltrat radiologique nouveau et persistant, associé au moins un signe clinique et/ou biologique d'infection (température $\geq 38,5$ ou $\leq 36,5$, hyperleucocytose, leuco-thrombopénie, PCT élevée), des secrétions purulentes, et un prélèvement respiratoire quantitatif proximal ou distal positif au seuil usuel. Seul le premier épisode de PAVM a été pris en compte. La PAVM est considérée comme précoce si rencontrée avant le $5^{\mathrm{e}}$ jour de ventilation, et tardive si survenue après le $5^{\mathrm{e}}$ jour. Les résultats sont exprimés en $\%$, moyenne \pm déviation standard, test du chi deux et test de MannWhitney, $\mathrm{p}<0,05$ significatif.

Résultats : Durant la période d'étude, 367 patients ont été hospitalisés. 71 patients $(19 \%)$ ont répondu aux critères d'inclusions, parmi lesquels $42 \%$ des cas $(n=30)$ ont développé une PAVM. L'âge moyen de notre population était de $39 \pm 15$ ans, avec une prédominance masculine (sex-ratio à 4), et la surface corporelle brûlée moyenne était de $42 \pm 18 \%$. Le délai moyen d'apparition de la PAVM a été de 8,5 jours [3-24 j]. La PAVM était précoce dans $16,6 \%$ et tardive dans $25,4 \%$. Les facteurs de risque de survenue de PAVM sont : le sexe masculin, l'atteinte de la face et la profondeur des lésions cutanées. Le profil bactériologique des PAVM était prédominant par l'Acinetobacter baumanii (26,6\%) et Klebsiella pneumoniae (20\%), puis Pseudomonas aeruginosa (10\%). Une bithérapie probabiliste, contenant essentiellement un pénème dans 21 cas $(70 \%)$ associé à un aminoside, était efficace et adaptée dans $70 \%$ des cas. La médiane de durée du traitement était de 8 jours. La durée moyenne de ventilation mécanique était de 19 jours vs 13 jours dans le groupe PAVM-, avec une durée de séjour en réanimation de $23 \pm 15$ jours. La mortalité globale en présence de PAVM était de $60 \%$.

Conclusion : La PAVM est fréquente chez les brûlés en réanimation avec une incidence de $8 \%$, et une prédominance des bacilles Gram négatifs multirésistants. L'importante morbimortalité associée à cette pathologie soulève plusieurs problèmes ; le délai et les outils diagnostics, le choix de l'antibiothérapie et les moyens de prévention à mettre en œuvre.

\section{EP015}

\section{Profil bactériologique des pneumopathies précoces} acquises sous ventilation mécanique

A. M'Rad ${ }^{1}$, N. Brahmi ${ }^{1}$, I. Fathallah ${ }^{1}$, I. Sedghiani ${ }^{1}$, Y. Blel $^{1}$, N. Kouraichi $^{1}$, H. El Ghord ${ }^{1}$, H. Thabet ${ }^{2}$, M. Amamou ${ }^{1}$

${ }^{1}$ Service de réanimation médicale polyvalente et toxicologique, centre d'assistance médicale-urgente (Camu), Tunis, Tunisie

${ }^{2}$ Service des urgences, centre d'assistance médicale-urgente (Camu), Tunis, Tunisie

Introduction : Les pneumopathies acquises sous ventilation mécanique $(\mathrm{PAVM})$ représentent un véritable problème de santé publique puisqu'elles sont à l'origine d'une augmentation de la durée de séjour et de ventilation assistée, d'un surcoût et d'une surmortalité propre de l'ordre de $20 \%$ [1,2]. Le but de ce travail est d'identifier les bactéries responsables des PAVM précoces ainsi que leurs profils de résistances vis à vis des différentes familles d'antibiotiques dans un service de réanimation médicale en Tunisie.

Patients et méthodes : étude prospective effectuée au service de réanimation médicale du CAMU sur une période de 24 mois de Janvier 2010 à décembre 2011. Tous les patients suspects de développer une PAVM précoce ont bénéficié d'un prélèvement distal protégé (PDP) par Combicath avec Culture quantitative. Le diagnostic de PAVM était retenu lorsque la culture est positive à un dénombrement de germes $\geq 10^{3}$ UFC. Nous avons considéré un délai de 7 jours pour la PAVM précoce.

Résultats : 50 patients suspects d'une PAVM précoce étaient inclus, 24 hommes et 26 femmes. La moyenne d'âge était de 35,5 \pm 14 ans. L'IGS 2 moyen, à l'admission était de $37,5 \pm 6$ et l'APACHE 2 moyen à $17,5 \pm 3$ avec une durée moyenne de ventilation mécanique à l'inclusion de 4,7 $\pm 1,6$ jours. Le diagnostic de la PAVM a été confirmé chez $64 \%(n=32)$ des patients. L'infection était uni-microbienne dans $69 \%(\mathrm{n}=22)$ des cas et bi-microbienne dans $31 \%(\mathrm{n}=10)$ des cas. L'A. baumannii était le germe le plus fréquent, retrouvé chez $84 \%$ $(\mathrm{n}=27)$ des patients ; suivi par le $P$. aeruginosa dans $44 \%(\mathrm{n}=14)$ des 


\begin{tabular}{|c|c|c|c|c|c|c|c|c|c|c|c|c|c|}
\hline \multirow[t]{2}{*}{ Bactéries } & \multicolumn{13}{|c|}{ Pourcentage de résistance aux antibiotiques } \\
\hline & TIC & $\mathrm{TCC}$ & PIP & $\mathrm{TZP}$ & CAZ & IMP & GM & AN & NT & $\mathrm{CS}$ & CIP & FOS & RA \\
\hline A. baumannii & 100 & 100 & 100 & 100 & 100 & 100 & 100 & 85 & 26 & 0 & 100 & 85 & 60 \\
\hline P. aeruginosa & 77 & 55 & 44 & 0 & 67 & 44 & 77 & 11 & 0 & 0 & 48 & 11 & - \\
\hline
\end{tabular}

cas et les entérobactéries chez $22 \%(\mathrm{n}=7)$ des patients. Parmi ces entérobactéries, la Klebsielle pneumoniae occupait le premier rang $(\mathrm{n}=3)$, suivie d'E. coli, du P. mirabilis, de P. stuartii et la S. Marcescens, chacune dans un cas. Pour les infections pluri microbiennes, l'association A. baumannii et $P$. aeruginosa était notée chez 6 patients (16\%), A. baumannii et entérobactérie chez 3 patients et $P$. aeruginosa et entérobactérie dans 2 cas. Dans $71 \%$, les entérobactéries étaient sécrétrices de ß-lactamase à spectre étendu (BLSE); les 2 autres étaient sécrétrices de pénicillinase de bas niveau de résistance. Le $P$. aeruginosa était résistant à la céftazidime et à l'imipénème dans $44 \%$ des cas. L'A. baumannii était résistant à toutes les bétalactamines dans tous les cas (Tableau 1).

Conclusion : A l'encontre des données de la littérature, le profil bactériologique des pneumopathies précoces n'est différent des PAVM tardives ni en terme des bactéries en cause ni en terme de résistances aux différents antibiotiques.

\section{Références}

1. Fagon JY, Chastre J, Vuagnat A, et al (1996) Nosocomial pneumonia and mortality among patients in intensive care units. JAMA 275:866-9

2. Bercault N, Boulain T (2001) Mortality associated with ventilator-associated pneumonia in an adult intensive care unit: A prospective 135 matched-paired cohort study. Crit Care Med 29:2303-9

\section{EP016}

\section{PAVM : diminution de l'incidence et la résistance} aux antibiotiques Est-ce possible?

M. Landais ${ }^{1}$, C. Bretonnière ${ }^{1}$, S. Corvec ${ }^{2}$, D. Villers ${ }^{1}$, C. Guitton ${ }^{1}$

${ }^{1}$ Service de réanimation médicale polyvalente,

CHU de Nantes-Hôtel-Dieu, Nantes, France
${ }^{2}$ Laboratoire de bactériologie, CHU de Nantes-Hôtel-Dieu, Nantes, France

Objectif : L'objectif de ce travail est de comparer l'épidémiologie et le profil de résistance des bactéries responsables des PAVM sur deux périodes distinctes de 10 ans.

Patients et méthodes : Il s'agit d'une étude prospective, conduite au sein d'un service de réanimation universitaire sur la période de janvier 2011 à juin 2013. Pour chaque PAVM (LBA), ont été recueillis les éléments suivants : âge, sexe, immunodépression, IGS2, bactérie(s) responsables(s), délai de survenue, mortalité en réanimation. Ces données ont été comparées à celles d'un recueil identique recueilli également prospectivement sur la période de janvier 2001 à juin 2003.

Résultats : Nous avons documenté 78 épisodes de PAVM chez 74 patients, causées par 95 bactéries entre 2011 et 2013 (Période B) et 97 épisodes de PAVM chez 77 patients causées par 106 bactéries entre 2001 et 2003 (Période A). Entre les 2 périodes, la proportion de staphylococcus aureus méticilline résistant (SARM) et de Pseudomonas aeruginosa (PA) diminue sans pour autant que l'on note une émergence d'entérobactéries résistantes (BLSE ou céphalosporinase hyperproduite).

Discussion : La diminution des SARM et des $P A$ n'est pas accompagnée d'émergence d'entérobactéries résistantes. La documentation systématique, le suivi de l'écologie en temps réel, une politique restrictive, une désescalade systématique de l'antibiothérapie et un délai de survenue des PAVM significativement plus court dans le groupe B peuvent être des facteurs favorisant. Existe t-il un environnement favorable dans notre CHU? Dans notre région?

Conclusion : L'écologie du service semble avoir évolué en 10 ans favorablement contrairement aux données de la littérature. Une maitrise de

\begin{tabular}{|c|c|c|c|c|c|c|c|c|c|}
\hline & \multirow[b]{2}{*}{ PA } & \multicolumn{3}{|c|}{ Entérobactéries } & \multicolumn{5}{|l|}{ S. Aureus } \\
\hline & & Total & BLSE & Case & SAMS & SARM & Pneumo. & H. Influ. & $\mathbf{n}$ \\
\hline A & $45(42 \%)$ & $16(15 \%)$ & 0 & 1 & $23(21,5 \%)$ & $5(5 \%)$ & $2(2 \%)$ & $8(7,5 \%)$ & 106 \\
\hline B & $20(21 \%)$ & $21(22 \%)$ & 0 & 1 & $23(24 \%)$ & 0 & $8(8,5 \%)$ & $9(9,5 \%)$ & 95 \\
\hline
\end{tabular}

\begin{tabular}{|llll|}
\hline & Période A (2001-2003) & Période B (2011-2013) & $p$ \\
\hline Admissions & 1940 & 2209 & 0,31 \\
Patients PAVM & 77 & 74 & 78 \\
Épisodes PAVM & 97 & $57[46-63]$ & 0,03 \\
Age (années) & $51[36-66]$ & $54 / 20$ & 0,117 \\
Ratio M/F & $61 / 16$ & $43[31-57,7]$ & 0,44 \\
IGSII & $46[37-57]$ & $9 / 69$ & 0,59 \\
Décès (décédés/vivants) & $23 / 74$ & $6[4-10]$ & 0,049 \\
Délai PAVM (j) & $10[5-20]$ & $15 / 63$ & 0,001 \\
Immunodépression (OUI/NON) & $6 / 69$ & & 0,04 \\
\hline
\end{tabular}


l'émergence des résistances semble possible. Néanmoins, les facteurs expliquant ces résultats nécessitent des investigations complémentaires.

\section{EP017}

Facteurs associés à la transition de la trachéobronchite acquise sous ventilation mécanique vers la pneumonie acquise sous ventilation mécanique

S. Nseir ${ }^{1}$, I. Martin-Loeches ${ }^{2}$, D. Makris ${ }^{3}$, A. Artigas ${ }^{2}$

${ }^{1}$ Pôle de réanimation, CHRU de Lille, Lille, France

${ }^{2}$ Réanimation polyvalente, Tauli Univ Hosp, Sabadell, Espagne

${ }^{3}$ Réanimation polyvalente, Larissa Univ Hosp, Larissa, Grèce

Introduction : Deux études randomisées récentes portant sur un nombre limité de patients ont suggéré que l'antibiothérapie pourrait être bénéfique chez les patients atteints de trachéobronchite acquise sous ventilation mécanique (TAVM). L'objectif de cette étude est de déterminer les facteurs associés à la transition de la TAVM vers la pneumonie acquise sous ventilation mécanique (PAVM).

Patients et méthodes : Cette étude prospective observationnelle a été réalisée dans 3 services de réanimation en Europe (Espagne, Grèce et France). Tous les patients présentant un premier épisode de TAVM étaient éligibles. Les patients trachéotomisés à l'admission et ceux qui ont développé une PAVM avant la TAVM ont été exclus. La TAVM a été définie par l'apparition de tous les critères suivants chez un patient sous ventilation mécanique depuis $>48 \mathrm{~h}$ : fièvre $\left(>38^{\circ} \mathrm{C}\right)$ en l'absence de toute autre cause, aspirations trachéales purulentes, culture positive de l'ECBT $\left(\geq 10^{5} \mathrm{cfu} / \mathrm{mL}\right)$ et absence de nouvel infiltrat à la radiographie thoracique. Le diagnostic de PAVM était basé sur des critères cliniques, radiologiques (nouvel infiltrat pulmonaire) et microbiologiques (ECBT $\geq 10^{5} \mathrm{cfu} / \mathrm{mL}$ ou LBA $\geq 10^{4} \mathrm{cfu} / \mathrm{mL}$ ). Seules les PAVM diagnostiquées dans les $96 \mathrm{~h}$ suivant le diagnostic de TAVM et causées par le même microorganisme ont été prises en compte. L'antibiothérapie était laissée à la discrétion des médecins. Les facteurs de risque de transition de la TAVM vers la PAVM ont été déterminés par analyse univariée et multivariée.

Résultats : 1710 patients intubés et ventilés $>48$ h étaient éligibles. $86(5,1 \%)$ patients ont été exclus pour trachéotomie à l'admission et $123(7,1 \%)$ pour une PAVM avant la TAVM. 122 patients ont présenté une TAVM, représentant $8,1 \%$ des 1501 patients restants. $17(13,9 \%)$ patients ont développé une PAVM ultérieure à la TAVM. Les microorganismes le plus souvent isolés étaient : $P$. aeruginosa (30\%), S. aureus (18\%) et $A$. baumannii (10\%). $13 \%$ des épisodes de TAVM étaient polymicrobiens. 74 (60\%) patients ont reçu une antibiothérapie. 58 (51\%) patients ont reçu une antibiothérapie appropriée. Le pourcentage des patients qui ont reçu une antibiothérapie appropriée était significativement plus élevé (53\% versus $11 \%, \mathrm{p}=0,002$ ) chez les patients qui n'ont pas présenté de PAVM ultérieure à la TAVM comparés à ceux qui en ont présenté une. Par contre le score SOFA (médiane $\left[25^{\mathrm{e}}, 75^{\mathrm{e}}\right.$ centiles] $4[3,7]$ vs $\left.6[6,9], \mathrm{p}=0,029\right)$ et les leucocytes $(10,6[7,2-14,6]$ vs $13,4[10,18,9], p=0,034)$ étaient significativement moins élevés chez ces deux groupes. L'analyse multivariée a identifié l'antibiothérapie appropriée comme seul facteur indépendamment associé au risque de transition de la TAVM vers la PAVM (OR [IC à $95 \%]: 0,12$ [0,02-0,59], $\mathrm{p}=0,009)$.

Conclusion : L'antibiothérapie appropriée est indépendamment associée à une diminution du risque de transition de la TAVM vers la PAVM.

\section{Bibliographie}

1. Nseir S, Favory R, Jozefowicz E, et al (2008) Antimicrobial treatment for ventilator-associated tracheobronchitis: a randomized, controlled, multicenter study. Crit Care 12:R62
2. Palmer LB, Smaldone GC, Chen JJ, et al (2008) Aerosolized antibiotics and ventilator-associated tracheobronchitis in the intensive care unit. Crit Care Med 36:2008-13

\section{EP018}

Suspicion de pneumopathie en réanimation : l'examen direct est-il utile pour la prescription de l'antibiothérapie probabiliste?

G. Gastaldi ${ }^{1}$, P. Gouin ${ }^{1}$, N. Frébourg ${ }^{2}$, E. Audureau ${ }^{3}$, A. Cerdan ${ }^{1}$, B. Dureuil ${ }^{1}$, B. Veber $^{1}$

${ }^{\text {}}$ Service d'anesthésie-réanimation Samu, CHU de Rouen, Rouen, France

${ }^{2}$ Département de microbiologie, $\mathrm{CHU}$ de Rouen, Rouen, France ${ }^{3}$ Pôle recherche clinique-santé publique, CHU Henri-Mondor, Créteil, France

Introduction : La pneumopathie est une cause fréquente de dégradation respiratoire au cours du séjour en réanimation. Les éléments cliniques et paracliniques ne permettent pas toujours d'affirmer avec certitude ce diagnostic. Notre objectif est de déterminer la fiabilité de l'examen direct $(E D)$ du prélèvement bronchique distal protégé (PBDP) pour le diagnostic des pneumopathies des patients de réanimation chirurgicale et la prescription d'une antibiothérapie.

Patients et méthodes : Étude prospective, observationnelle, monocentrique. Patients sous ventilation mécanique, hospitalisés en réanimation chirurgicale, présentant une suspicion clinique de pneumopathie et devant bénéficier d'un PBDP sous fibroscopie. Le prélèvement a été acheminé au laboratoire, où ont été effectués un ED par coloration Gram, et une culture quantitative. Le diagnostic de pneumopathie était retenu par le clinicien sur des critères cliniques, radiologiques et bactériologiques. Les résultats de l'ED ont été comparés au diagnostic de pneumopathie et exprimés sous forme de sensibilité (Se), spécificité (Sp), valeurs prédictives positive et négative (VPP et VPN). La concordance entre l'ED et la culture a été analysée par le test de corrélation kappa (k). Les caractéristiques de l'antibiothérapie ont été étudiées (délais d'instauration, délais d'efficacité, durée de traitement).

Résultats : De mai 2011 à juin 2012, 80 PBDP ont été réalisés chez 66 patients (IGS-2 moyen : 47,9 $\pm 16,9$ ). Le diagnostic de pneumopathie a été retenu dans 45 cas. L'ED était positif dans 29 cas, dont 3 faux positifs. La Se était de $57,8 \%$, la Sp de $91,4 \%$, la VPP de $89,7 \%$, et la VPN de $62,7 \%$. Le $\mathrm{k}$ était à 0,40 . Une antibiothérapie efficace a été instaurée dans les 24 heures suivant la réalisation du PBDP dans $73,3 \%$ des cas de pneumopathie. Le retard à l'efficacité thérapeutique était dû à la négativité de l'ED dans $8,9 \%$ des pneumopathies.

Conclusion : L'ED du PBDP ne semble pas fiable pour le dépistage des pneumopathies du patient sous ventilation mécanique (Faible VPN). Ces résultats sont relativement superposables à ceux de la littérature. En revanche, du fait de sa forte VPP, sa positivité devrait inciter le praticien à introduire une antibiothérapie probabiliste, dont le choix ne peut être guidé par le détail du Gram (faible k).

\section{EP019}

Performance et impact de l'antibiogramme réalisé directement à partir des prélèvements respiratoires profonds pour le diagnostic des pneumonies acquises sous ventilation mécanique (PAVM)

M. Le Dorze ${ }^{1}$, N. Gault ${ }^{2}$, A. Foucrier' ${ }^{1}$, E. Ruppé $e^{3}$, P.-L. Woerther ${ }^{3}$, B. Mourvillier', P. Montravers', M.-P. Dilly ${ }^{4}$, A. Andremont ${ }^{3}$, M. Wolff', L. Armand-Lefèvre ${ }^{3}$ 
${ }^{1}$ Service de réanimation médicale et infectieuse,

CHU Bichat-Claude-Bernard, Paris, France

${ }^{2}$ Épidémiologie et recherche clinique, CHU Bichat-Claude-Bernard,

Paris, France

${ }^{3}$ Laboratoire de bactériologie, CHU Bichat-Claude-Bernard,

Paris, France

${ }^{4}$ Département d'anesthésie et réanimation,

CHU Bichat-Claude-Bernard, Paris, France

Introduction : Une antibiothérapie probabiliste inadaptée est associée à une augmentation de la mortalité des PAVM. Si une antibiothérapie probabiliste à large spectre est recommandée, elle doit être suivie d'une désescalade dès le rendu de l'antibiogramme (48 h), afin de limiter l'émergence de bactéries résistantes. L'antibiogramme direct (ATBD), réalisé directement à partir du prélèvement clinique, peut être rendu dès $24 \mathrm{~h}$. L'objectif était d'évaluer les performances de l'ATBD par rapport à l'antibiogramme standard (ATBS) et son impact potentiel chez les patients atteints de PAVM.

Patients et méthodes : Pendant 18 mois, dans 3 réanimations, un ATBD a été réalisé sur les LBA (lavage broncho-alvéolaire) et PDP (prélèvement distal protégé) des patients suspects de PAVM et présentant des bacilles à Gram négatifs à l'examen direct. Les ATBD et ATBS ont été réalisés par diffusion en milieu gélosé et les identifications par spectrométrie de masse. Dans les prélèvements polymicrobiens, la bactérie la plus résistante a été prise en compte. Les sensibilités (Se), spécificités ( $\mathrm{Sp}$ ), valeurs prédictives positives (VPP) et négatives (VPN) de l'ATBD comparé à l'ATBS ont été calculées. Les données cliniques et d'antibiothérapie ont été collectées.

Résultats : 80 patients ont été inclus (âge : $63 \pm 16$ ans, sex-ratio : $2,7$, IGS II : $53 \pm 19)$. 73/85 (86 \%) prélèvements ont pu être analysés. Les performances de l'ATBD sont présentées dans le tableau. Les prélèvements étaient monomicrobiens dans $58(80 \%)$ cas, $15(20 \%)$ contenaient 2 espèces. Les bactéries en cause étaient 48 entérobactéries $(55 \%$, E. coli $\mathrm{n}=15)$ et 40 non fermenteurs $(45 \%$, P. aeruginosa $\mathrm{n}=33)$. Les antibiothérapies ont été recueillies pour 66 épisodes : dans 6 cas $(9 \%)$, l'antibiothérapie initiale était inadaptée, et dans 22 cas (33\%), une désescalade était possible. Une optimisation de l'antibiothérapie dès $24 \mathrm{~h}$ aurait été possible dans $42 \%$ des épisodes et 15 jours de carbapénèmes auraient pu être économisés.

Conclusion : La performance diagnostique de l'antibiogramme direct est excellente. Cet outil pourrait permettre une optimisation $24 \mathrm{~h}$ plus tôt de l'antibiothérapie dans $40 \%$ des PAVM : antibiothérapie initiale efficace et désescalade plus précoce.

\section{Bibliographie}

1. Boyer A, Medrano J, Mzali F, et al (2012) Direct testing of bronchoalveolar lavages from ventilator-associated pneumonia patients. Diagn Microbiol Infect Dis 73:107-10

\begin{tabular}{|lllll|}
\hline Antibiotique testé & $\begin{array}{l}\text { Se } \\
\mathbf{( \% )}\end{array}$ & $\begin{array}{l}\text { Sp } \\
\mathbf{( \% )}\end{array}$ & $\begin{array}{l}\text { VPP (\%) } \\
\text { Prédit } \\
\text { la résistance }\end{array}$ & $\begin{array}{l}\text { NPV (\%) } \\
\text { Prédit } \\
\text { la sensibilité }\end{array}$ \\
\hline Ticarcilline & 100 & 95,7 & 98 & 100 \\
Piperacilline & 100 & 96,3 & 97,9 & 100 \\
Piperacilline-Tazobactam & 100 & 97,5 & 97,1 & 100 \\
Ceftazidime & 100 & 93,8 & 89,3 & 100 \\
Imipeneme & 100 & 96,3 & 90,5 & 100 \\
Amikacine & 88,9 & 96,4 & 88,9 & 96,4 \\
Ciprofloxacine & 100 & 95 & 94,3 & 100 \\
\hline
\end{tabular}

2. Leone M, Garcin F, Bouvenot J, et al (2007) Ventilator-associated pneumonia: breaking the vicious circle of antibiotic overuse. Crit Care Med 35:379-85

\section{EP020 \\ Épiglottites aiguës de l'adulte hospitalisées en réanimation : à propos de 34 cas}

T. Chroboczek ${ }^{1}$, M. Cour ${ }^{1}$, T. Baudry ${ }^{1}$, M. Simon ${ }^{1}$, J. Turc $^{1}$,

E. Faucher ${ }^{1}$, R. Hernu ${ }^{1}$, G. Petit ${ }^{1}$, B. Allaouchiche ${ }^{2}$, J. Bohe $^{3}$,

L. Argaud ${ }^{1}$

${ }^{1}$ Service de réanimation médicale,

CHU de Lyon-hôpital Édouard-Herriot, Lyon, France

${ }^{2}$ Service de réanimation chirurgicale,

CHU de Lyon-hôpital Édouard-Herriot, Lyon, France

${ }^{3}$ Service de réanimation médicale, CHU de Lyon-CH Lyon Sud,

Pierre-Bénite, France

Introduction : L'épiglottite aiguë est une maladie infectieuse rare, d'incidence croissante chez l'adulte, pouvant mettre en jeu le pronostic vital par obstruction des voies aériennes supérieures. Néanmoins, elle a été peu étudiée en réanimation.

Patients et méthodes : Tous les cas d'épiglottites aiguës de l'adulte hospitalisés dans 5 services de réanimation entre 1999 et 2013 ont été rétrospectivement recensés. Ont été recueillies des données cliniques, biologiques, radiologiques, thérapeutiques ainsi que l'évolution. Les patients ayant bénéficié d'une ventilation artificielle invasive ont été comparés aux patients non intubés.

Résultats : Les données de 34 patients ont été analysées : âge $44 \pm 13$ ans ; sexe ratio 5,8; IGS2 $24 \pm 16$. Seize patients (47\%) étaient fumeurs, 8 (24\%) n'avaient aucun antécédent. Le temps d'évolution des symptômes avant l'admission en réanimation était de $66 \pm 86$ heures. Les deux principaux motifs d'hospitalisation en réanimation étaient la nécessité d'une surveillance $(\mathrm{n}=17,50 \%)$ ou une détresse respiratoire aiguë $(\mathrm{n}=10,29 \%)$. Les symptômes les plus fréquents étaient : douleur de gorge $(\mathrm{n}=30,88 \%)$, dysphagie $(\mathrm{n}=29,85 \%)$, dyspnée $(\mathrm{n}=26$, $76 \%)$, et dysphonie $(\mathrm{n}=22,65 \%)$. Un abcès était objectivé en TDM dans 13 cas $(38 \%)$. Une étiologie microbiologique était documentée chez seulement 9 patients ( $26 \%$ ), avec un Streptococcus spp isolé dans 7 cas $(21 \%)$. Treize patients $(38 \%)$ ont eu recours à une ventilation mécanique invasive. L'intubation trachéale a été réalisée chez un patient vigile à l'aide d'un naso-fibroscope dans 9 cas (26\%). Une trachéotomie a été réalisée en urgence dans 2 cas $(6 \%) ; 11$ patients $(32 \%)$ ont nécessité une prise en charge chirurgicale. Deux patients $(6 \%)$ sont décédés, chaque fois après un arrêt cardiaque hypoxiques avant intubation trachéale. Les durées moyennes de séjour en réanimation et à l'hôpital étaient respectivement de $6 \pm 8$ et $12 \pm 12$ jours. En analyse univariée, le délai entre le début des symptômes et l'admission en réanimation était significativement plus long chez les patients pour lesquels une ventilation artificielle a été nécessaire $(\mathrm{p}<0,05)$. Même si la gravité des deux groupes de patients était assez superposable, l'existence d'une pneumonie communautaire associée était observée chez 3 (23\%) des patients ventilés (aucun dans l'autre groupe, $\mathrm{p}<0,05$ ). Une documentation microbiologique a été obtenue 6 fois $(46 \%)$ dans le groupe de patients ventilés ( 5 fois à Streptoccus spp.) versus seulement 2 fois $(10 \%)$ dans l'autre groupe $(\mathrm{p}=0,001)$.

Conclusion : Dans ce travail, le profil type des cas d'épiglottites admis en réanimation est représenté par des hommes jeunes, fumeurs, ayant pas ou peu de comorbidité. Une infection à Streptococcus spp est l'étiologie la plus fréquemment retrouvée. L'évolution est favorable si une protection de la filière respiratoire a pu être réalisée dans de bonnes conditions de sécurité. 